\title{
Placental mitochondrial DNA
} and CYP1A1 gene methylation as molecular signatures for tobacco smoke exposure in pregnant women and the relevance for birth weight

Bram G. Janssen ${ }^{1}$, Wilfried Gyselaers ${ }^{2,3}$, Hyang-Min Byun ${ }^{4}$, Harry A. Roels ${ }^{1,5}$, Ann Cuypers ${ }^{1}$, Andrea A. Baccarelli ${ }^{4,6}$ and Tim S. Nawrot ${ }^{1,7,8^{*}}$

\begin{abstract}
Background: Maternal smoking during pregnancy results in an increased risk of low birth weight through perturbations in the utero-placental exchange. Epigenetics and mitochondrial function in fetal tissues might be molecular signatures responsive to in utero tobacco smoke exposure.

Methods: In the framework of the ENVIRONAGE birth cohort, we investigated the effect of self-reported tobacco smoke exposure during pregnancy on birth weight and the relation with placental tissue markers such as, (1) relative mitochondrial DNA (mtDNA) content as determined by real-time quantitative PCR, (2) DNA methylation of specific loci of mtDNA (D-loop and MT-RNR1), and (3) DNA methylation of the biotransformation gene CYP1A1 (the last two determined by bisulfite-pyrosequencing). The total pregnant mother sample included 255 non-smokers, 65 formersmokers who had quit smoking before pregnancy, and 62 smokers who continued smoking during pregnancy.
\end{abstract}

Results: Smokers delivered newborns with a birth weight on average $208 \mathrm{~g}$ lower [95\% confidence interval (Cl) -318 to $-99, p=0.0002$ ] than mothers who did not smoke during pregnancy. In the smoker group, the relative mtDNA content was lower $(-21.6 \%, 95 \% \mathrm{Cl}-35.4$ to $-4.9 \%, p=0.01)$ than in the non-smoker group; whereas, absolute mtDNA methylation levels of MT-RNR1 were higher $(+0.62 \%, 95 \% \mathrm{Cl} 0.21$ to $1.02 \%, p=0.003)$. Lower CpG-specific methylation of CYP1A1 in placental tissue $(-4.57 \%, 95 \% \mathrm{Cl}-7.15$ to $-1.98 \%, p<0.0001)$ were observed in smokers compared with non-smokers. Nevertheless, no mediation of CYP1A1 methylation nor any other investigated molecular signature was observed for the association between tobacco smoke exposure and birth weight.

Conclusions: mtDNA content, methylation of specific loci of mtDNA, and CYP1A1 methylation in placental tissue may serve as molecular signatures for the association between gestational tobacco smoke exposure and low birth weight.

Keywords: Birth weight, CYP1A1, Epigenetics, DNA methylation, Mitochondrial DNA content, Mitochondrial DNA methylation, Placental tissue, Tobacco smoke

\footnotetext{
*Correspondence: Tim.Nawrot@uhasselt.be

${ }^{8}$ Centre for Environmental Sciences, Hasselt University, Agoralaan

Gebouw D, 3590 Diepenbeek, Belgium

Full list of author information is available at the end of the article
} 


\section{Background}

A growing area of research interest with major public health implications are the consequence of insults during fetal life for the health status in child- and adulthood. It is well known that maternal smoking during pregnancy increases the risk of low birth weight $[1,2]$ and preterm delivery $[3,4]$ which is probably due to perturbations in the fetoplacental exchange [5]. The exact mechanism(s) underlying these adverse effects remain unclear, but emerging data suggests that biochemical, genetic, and epigenetic processes respond to and/or are modified by in utero tobacco exposure of the fetal organism.

Tobacco smoke consists of particulate and gaseous phases containing more than 7000 chemicals of which at least 70 substances are known to cause cancer [6]. Constituents of tobacco smoke such as polycyclic aromatic hydrocarbons (PAHs) enter cells and may activate genes involved in detoxification processes such as CYP1A1 (cytochrome $\mathrm{P} 450$, family 1 , subfamily A, polypeptide 1) via the aryl hyrdrocarbon receptor (Ahr) signaling pathway resulting in an oxidative imbalance of the cells. Mitochondrial DNA (mtDNA), which resides as multiple double stranded circular copies in mitochondria, is extremely vulnerable and responsive to tobaccoinduced oxidative stress [7-9]. As a result, alterations in mtDNA content, characterized as increasing or decreasing mtDNA copies, are an indication of dysfunctional or damaged mitochondria [10]. The inter-genomic crosstalk between mitochondria and the nucleus is complex. Growing evidence suggests that mitochondrial dysfunction may affect the epigenetic landscape of the nuclear genome $[11,12]$. DNA methylation is the most intensively studied epigenetic modification. Exposures to adverse environmental factors are important determinants for methylation programming during early life [13, 14]. Global [15-18] and gene-specific (e.g. CYP1A1) [19-26] DNA methylation differences have been demonstrated in cord blood and placental cells of neonates from mothers who smoked during pregnancy. Disruption of the fetal methylome has been associated with adverse pregnancy outcomes and could provide an underlying mechanism through which smoking affects fetal growth [20, 24, 27].

While several studies described separately the effect of maternal smoking during pregnancy on birth weight, mitochondrial DNA, and CYP1A1 methylation, we integrated these biological endpoints in our investigation of placental tissue collected in the framework of the ENVIRONAGE birth cohort study [28]. We hypothesized that exposure to tobacco smoke during pregnancy impacts birth weight and concomitantly also these molecular signatures.

\section{Methods}

\section{Study population}

In the present study, 382 mother-newborn pairs were enrolled in the ENVIRONAGE birth cohort in Belgium (acronym for ENVIRonmental influence ON AGEing in early life). All procedures were approved by the Ethical Committee of Hasselt University and East-Limburg Hospital. The study design and procedures were previously described in detail [29]. Briefly, written informed consent was obtained from each participating mother who gave birth in the East-Limburg Hospital in Genk, Belgium. For this study, the only inclusion criterion was that mothers had to be able to fill out questionnaires in Dutch. Enrolment was equally spread over all seasons of the year. Questionnaires and medical records were consulted after birth and provided information on maternal age, maternal education, smoking status, ethnicity, pre-pregnancy body mass index (BMI), gestational age, newborn's sex, Apgar scores, birth weight and length, parity, and ultrasonographic data. Maternal education was coded as "low" (no diploma or primary school), "middle" (high school) or "high" (college or university degree). Based on the native country of the newborn's grandparents we classified his/her ethnicity as European-Caucasian when two or more grandparents were European, or non-European when at least three grandparents were of non-European origin. We asked the mothers whether they consumed alcohol during pregnancy, used medication, and how many times per week they practiced physical exercises for at least $20 \mathrm{~min}$. Information about tobacco smoke exposure was collected by self-report of the mothers. They were asked whether they continued smoking during pregnancy (smoker group, $n=62$ ), whether they smoked before pregnancy and stopped when pregnant (pastsmoker group, $n=65$ ), or whether they never smoked in their life (non-smoker group, $n=255$ ). Mothers who had ever smoked filled out the number of smoking years and the number of cigarettes smoked per day before and during pregnancy. We also asked the mothers how long (months) they continued smoking before becoming aware of being pregnant. Furthermore, we have data on passive smoke exposure (due to indoor smoking by somebody else).

\section{Sample collection}

Placentas were deep-frozen within 10 min after delivery. Specimens of placental tissue were taken on minimally thawed placentas for DNA extraction. We took villous tissue $\left(1-2 \mathrm{~cm}^{3}\right)$ at a fixed location from the fetal side of the placenta, approximately $1-1.5 \mathrm{~cm}$ below the chorio-amniotic membrane, and preserved the biopsies at $-80{ }^{\circ} \mathrm{C}[30]$. At a later stage, genomic DNA was isolated 
from the placental biopsies using the QIAamp DNA mini kit (Qiagen, Inc., Venlo, Netherlands) and stored at $-80{ }^{\circ} \mathrm{C}$ until further use.

\section{DNA methylation analysis}

We performed DNA methylation analysis by highly quantitative bisulfite polymerase chain reaction (PCR) pyrosequencing as previously described in detail [30]. Bisulfite conversions were performed using $1 \mu \mathrm{g}$ of extracted genomic DNA with the EZ-96 DNA methylation Gold kit (Zymo Research, Orange, CA, USA) according to the manufacturer's instructions. We examined four CpG sites within the promoter region of the CYP1A1 gene and for the mitochondrial genome we examined two CpG sites in the MT-RNR1 region, and three CpG sites in the D-loop region. Detailed information regarding primer sequences is given in Additional file 1: Table S1. Prior to pyrosequencing, PCR amplification of regions of interest was performed in a total reaction volume of $30 \mu \mathrm{l}$, containing $15 \mu \mathrm{l}$ GoTaq Hot Start Green Master Mix (Promega, Madison, WI, USA), 10 pmol forward primer, $10 \mathrm{pmol}$ reverse primer, $1 \mu \mathrm{l}$ bisulfite-treated genomic DNA, and water. PCR products were purified and sequenced by pyrosequencing using the PyroMark Q96 MD Pyrosequencing System (Qiagen, Inc., Germantown, MD, USA). The degree of methylation was expressed as the ratio (percentage) of methylated cytosines over the sum of methylated and unmethylated cytosines. The efficiency of the bisulfite-conversion process was assessed using non-CpG cytosine residues within the sequence. We used 0\% (PSQ-T oligo: 5'-TTGCGATAC A A CG G GA ACAA ACG T TGAAT TC-3') and 100\% (PSQ-C oligo: 5'-TTGCGATACGACGGGAACAAACGTTGAATTC-3') DNA methylation control oligos. The sequencing primer for the control oligo was: 5'-AACGTTTGTTCCCGT-3'. We mixed the PSQ-C oligo (or PSQ-T oligo) with the sequencing oligo in PyroMark Annealing Buffer (Qiagen, Inc., Valencia, CA, USA) and performed pyrosequencing with the sequencing entry C/TGTAT. We assessed the within-placenta variability in a random subset of 19 placentas as previously described [30]. The between-placenta variability was higher than the within-placenta variability for CYP1A1 (58 vs. $42 \%$, $p<0.0001$ ), the $D$-loop region (61 vs. $39 \%, p=0.01$ ), and $M T-R N R 1$ ( 58 vs. $42 \%, p=0.009$ ).

\section{Mitochondrial DNA content analysis}

The mtDNA content was measured by determining the ratio of two mitochondrial gene copy numbers (MTF3212/R3319 and MT-ND1) to two single-copy nuclear control genes ( $R P L P O$ and $A C T B)$ using a quantitative real-time PCR (qPCR) assay as previously described [29] and used with a small modification.
Isolated genomic DNA (12.5 ng) was added to $7.5 \mu \mathrm{l}$ mastermix consisting of Fast SYBR ${ }^{\circledR}$ Green I dye $2 \mathrm{x}(5 \mu \mathrm{l} /$ reaction), forward and reverse primer (each $0.3 \mu \mathrm{l} /$ reaction), and RNase free water $(1.9 \mu \mathrm{l} /$ reaction) for a final volume of $10 \mu \mathrm{l}$ per well. Primer sequences (Additional file 1: Table S1) were diluted to a final concentration of $300 \mathrm{nM}$ in the master mix. Samples were run in triplicate in a 384-well format. Real-time PCR was performed using the 7900HT Fast Real-Time PCR System (Applied Biosystems, Foster City, CA, USA) with the following thermal cycling profile: $20 \mathrm{~s}$ at $95{ }^{\circ} \mathrm{C}$ (activation), followed by 40 cycles of $1 \mathrm{~s}$ at $95{ }^{\circ} \mathrm{C}$ (denaturation) and $20 \mathrm{~s}$ at $60^{\circ} \mathrm{C}$ (annealing/extension), and ending with melting curve analysis $\left(15 \mathrm{~s}\right.$ at $95^{\circ} \mathrm{C}, 15 \mathrm{~s}$ at $60^{\circ} \mathrm{C}, 15 \mathrm{~s}$ at $\left.95^{\circ} \mathrm{C}\right)$. qBase software (Biogazelle, Zwijnaarde, Belgium) was used to normalize data and correct for run-to-run differences [31].

\section{Statistical analysis}

We used SAS software (version 9.2; SAS Institute Inc., Cary, NC, USA) for database management and statistical analysis. Relative mtDNA content (unitless) was $\log _{10^{-}}$ transformed to normalize the distribution. The relationships between smoking and continuous variables were examined with one-way ANOVA procedures and Chi square tests for the categorical variables. We applied conventional multiple linear regression to estimate the association between maternal smoking status and birth weight, length, or placental mtDNA content. The pyrosequencing-based DNA methylation analysis produced a methylation value (\%) for each $\mathrm{CpG}$ site of CYP1A1 (four CpGs), MT-RNR1 (two CpGs) and the D-loop region (three $\mathrm{CpGs)}$. Correlations between adjacent $\mathrm{CpG}$ sites within one gene or region were tested with Pearson correlation coefficients. With mixed-effects models, we took into account each CpG dinucleotide position and tested the association between gene-specific DNA methylation and maternal smoking status. We applied Dunnett's test for multiple comparisons of smokers and past-smokers with the reference group (non-smokers). Maternal alcohol consumption, medication use, physical activity, maternal education, ethnicity, maternal age, pre-pregnancy BMI, parity, gestational age, and newborn's sex were considered as possible confounders, but only those associated with maternal smoking $(p \leq 0.05)$ and which potentially could influence birth weight and length, mtDNA content or DNA methylation were considered for entry in the models. However, newborn's sex, maternal age, gestational age, ethnicity, parity, and prepregnancy BMI were forced into the model regardless of the $p$ value, in addition to maternal education, and alcohol consumption. Q-Q plots of the residuals were used to test the linearity assumption of the models. 
In a sensitivity analysis, Pearson correlation coefficients were calculated between birth weight or length and measures of smoking (years of smoking, pack-year or number of cigarettes smoked per day during pregnancy). Furthermore, we used mediation analysis to investigate whether the examined molecular signatures underlie the association between gestational tobacco smoke exposure and birth weight [32].

\section{Results}

\section{Participant's demographics and lifestyle factors}

Demographic characteristics and perinatal factors of 382 mother-newborn pairs are reported in Table 1. The newborns, among them 194 girls $(50.8 \%)$, had a mean gestational age of 39.2 weeks (range 35-42) and comprised $200(52.3 \%)$ primiparous and $142(37.2 \%)$ secundiparous newborns. The mean (SD) birth weight of the newborns was 3429 (426) $\mathrm{g}$ and birth length $50.3(1.9) \mathrm{cm}$. About $90 \%(n=332)$ of the newborns were Europeans of Caucasian ethnicity. Mean maternal age was 29.0 years (range 18-42 years). Most women $(66.7 \%, n=255)$ never smoked cigarettes and 65 women (17.0\%) stopped smoking before pregnancy; whereas, 62 mothers (16.2\%) reported to have smoked during pregnancy [on average 7.8 cigarettes per day (inter quartile range, IQR: $5-10]$. A fair number of mothers ( $n=73,19.1 \%)$ occasionally consumed alcohol during their pregnancy.

Compared to the non-smokers, the group of smoking mothers were younger $(27.7 \pm 4.8$ vs. 29.0 years \pm 4.7 , $p=0.008)$, comprised less women with higher education ( 22.6 vs. $59.6 \%, p<0.0001$ ), and delivered newborns of lower birth weight and length. Alcohol consumption was higher in the past-smoker group than in the non-smoker group (30.8 vs. $16.5 \%, p=0.01$ ).

\section{Smoking status and birth parameters}

Birth weight and length were respectively $225 \mathrm{~g}$ and $1 \mathrm{~cm}$ lower for newborns from the smoker mothers compared to the non-smokers (Table 1). After adjustment for maternal age, gestational age, newborn's sex, maternal education, ethnicity, parity, pre-pregnancy BMI, and alcohol consumption, we still observed a lower birth weight $(-208 \mathrm{~g}$, $95 \% \mathrm{CI}-318$ to $-99 \mathrm{~g}, p=0.0002)$ and a shorter birth length $(-1.0 \mathrm{~cm}, 95 \% \mathrm{CI}-1.5$ to $-0.5 \mathrm{~cm}, p<0.0001)$ in newborns delivered by women who continued smoking during pregnancy compared to non-smoking mothers. Mothers who stopped smoking before pregnancy delivered newborns whose birth weight $(p=0.55)$ or length $(p=0.87)$ did not differ from that of never-smokers.

\section{Smoking status and mtDNA in placental tissue}

After adjustment for the aforementioned covariates, the relative mtDNA content in placental tissue was $21.6 \%$ (95\%
$\mathrm{CI}-35.4$ to $-4.9, p=0.01)$ lower in smoking mothers, but not in past-smokers $(p=0.72)$, in comparison with non-smokers (Fig. 1). In contrast, absolute methylation levels of the mitochondrial genome at the MT-RNR1 gene were higher in mothers who continued smoking during pregnancy $(+0.62 \%, 95 \%$ CI 0.21 to $1.02, p=0.003)$ and marginally higher in mothers who stopped smoking prior to pregnancy $(+0.37 \%, 95 \% \mathrm{CI}-0.02$ to $0.75, p=0.06)$ compared with non-smokers (Fig. 1). We found no interaction between smoking status and CpG site of $M T-R N R 1$ $\left(p_{\text {int }}=0.94\right)$, and the methylation levels at the D-loop region did not differ between the groups $(p=0.85)$.

\section{Smoking status and gene-specific CYP1A1 methylation in placental tissue}

The examined CpGs in the promoter region of CYP1A1 showed strong inter-correlations for placental tissue $(\mathrm{r}=0.71-0.93, p<0.0001)$ (Additional file 1: Figure S1). Unadjusted mixed-effects models revealed an interaction effect between smoking status and CpG sites of the promoter region of CYP1A1 ( $\left.p_{\text {int }}<0.0001\right)$. Placental methylation levels at CpG3 were significantly lower in mothers who continued smoking during pregnancy compared to non-smoking mothers (Fig. 2), even after adjustment for maternal age, gestational age, newborn's sex, maternal education, ethnicity, parity, pre-pregnancy BMI, and alcohol consumption $(-4.57 \%, 95 \% \mathrm{CI}-7.15$ to $-1.98, p<0.0001$ ) (Table 2). No significant differences in CpG methylation levels were observed in mothers who stopped smoking before pregnancy.

\section{Sensitivity analysis}

As anticipated, we observed a clear dose-effect relation between birth weight or length and measures of smoking status (years of smoking, pack-year, or the number of cigarettes smoked per day during pregnancy). In comparison with non-smokers, no significant difference was observed in birth weight or length of newborns from mothers who stopped smoking for a longer period of time before pregnancy or mothers who stopped just prior to pregnancy. We observed a positive association of CYP1A1 methylation levels with placental mtDNA content $(\mathrm{r}=0.14, p=0.005)$, and a negative association with placental mtDNA methylation $(\mathrm{r}=-0.11, p=0.02)$ (Fig. 3). Furthermore, we observed no mediation of CYP1A1 methylation nor any other investigated molecular signature between the association of tobacco smoke exposure and birth weight (data not shown).

\section{Discussion}

The present investigation showed that women who smoked during pregnancy had neonates with lower birth weight and length, lower mtDNA content, higher 
Table 1 Characteristics of mother-newborn pairs according to self-reported tobacco smoke exposure during pregnancy

\begin{tabular}{|c|c|c|c|c|c|}
\hline Variable & $\begin{array}{l}\text { All } \\
(n=382)\end{array}$ & $\begin{array}{l}\text { Non-smokers } \\
(n=255)\end{array}$ & $\begin{array}{l}\text { Past-smokers } \\
(n=65)\end{array}$ & $\begin{array}{l}\text { Smokers } \\
(n=62)\end{array}$ & $p$ value* \\
\hline \multicolumn{6}{|l|}{ Newborn } \\
\hline Sex & & & & & 0.47 \\
\hline Male & $188(49.2 \%)$ & $120(47.1 \%)$ & $34(52.3 \%)$ & $34(54.8 \%)$ & \\
\hline Female & $194(50.8 \%)$ & $135(52.9 \%)$ & $31(47.7 \%)$ & $28(45.2 \%)$ & \\
\hline Ethnicity & & & & & 0.73 \\
\hline European-Caucasian & $332(86.9 \%)$ & $223(87.4 \%)$ & $57(87.7 \%)$ & $52(83.9 \%)$ & \\
\hline Non-European & $50(13.1 \%)$ & $32(12.6 \%)$ & $8(12.3 \%)$ & $10(16.1 \%)$ & \\
\hline Gestational age, w & $39.2 \pm 1.2$ & $39.3 \pm 1.2$ & $39.2 \pm 1.3$ & $39.2 \pm 1.2$ & 0.82 \\
\hline Birth weight, $g$ & $3429 \pm 426$ & $3472 \pm 424$ & $3437 \pm 423$ & $3247 \pm 395$ & 0.0009 \\
\hline Birth length, $\mathrm{cm}$ & $50.3 \pm 1.9$ & $50.5 \pm 2.0$ & $50.5 \pm 1.7$ & $49.5 \pm 1.8$ & 0.0007 \\
\hline \multicolumn{6}{|l|}{ Mother } \\
\hline Age, year & $29.0 \pm 4.7$ & $29.4 \pm 4.5$ & $28.9 \pm 4.9$ & $27.7 \pm 4.8$ & 0.03 \\
\hline Pre-pregnancy BMI, kg/m² & $24.3 \pm 4.5$ & $24.2 \pm 4.4$ & $24.8 \pm 5.3$ & $24.2 \pm 4.1$ & 0.65 \\
\hline Maternal education & & & & & $<0.0001$ \\
\hline Low & $51(13.3 \%)$ & $26(10.2 \%)$ & $6(9.2 \%)$ & $19(30.6 \%)$ & \\
\hline Middle & $131(34.3 \%)$ & $77(30.2 \%)$ & $25(38.5 \%)$ & $29(46.8 \%)$ & \\
\hline High & $200(52.4 \%)$ & $152(59.6 \%)$ & $34(52.3 \%)$ & $14(22.6 \%)$ & \\
\hline Medication use ${ }^{a}$ & & & & & 0.57 \\
\hline None & $134(37.8 \%)$ & $92(39.5 \%)$ & $21(32.3 \%)$ & $21(37.5 \%)$ & \\
\hline Alcohol consumption & & & & & 0.03 \\
\hline Occasionally & $73(19.1 \%)$ & $42(16.5 \%)$ & $20(30.8 \%)$ & $11(17.7 \%)$ & \\
\hline Physical activity (>20 min) ${ }^{b}$ & & & & & 0.40 \\
\hline$<1$ times per week & $122(33.3 \%)$ & $82(33.3 \%)$ & $19(29.7 \%)$ & $21(36.8 \%)$ & \\
\hline 1 times per week & $86(23.4 \%)$ & $63(25.6 \%)$ & $15(23.4 \%)$ & $8(14.0 \%)$ & \\
\hline$>2$ times per week & $159(43.3 \%)$ & $101(41.1 \%)$ & $30(46.9 \%)$ & $28(49.2 \%)$ & \\
\hline Parity & & & & & 0.42 \\
\hline 1 & $200(52.3 \%)$ & $132(51.8 \%)$ & $36(55.4 \%)$ & $32(51.6 \%)$ & \\
\hline 2 & $142(37.2 \%)$ & $91(35.7 \%)$ & $25(38.5 \%)$ & $26(41.9 \%)$ & \\
\hline$\geq 3$ & $40(10.5 \%)$ & $32(12.5 \%)$ & $4(6.1 \%)$ & $4(6.5 \%)$ & \\
\hline Cigarettes before pregnancy & - & - & $11.0 \pm 6.9$ & $10.3 \pm 6.7$ & \\
\hline Cigarettes during pregnancy & - & - & - & $7.8 \pm 4.6$ & \\
\hline
\end{tabular}

Data are presented as arithmetic mean \pm standard deviation (SD) or number (\%)

* $p$ value derived from one-way ANOVA or Chi square tests in case of continuous or categorical variables respectively

${ }^{a}$ Medication use: occasional use of paracetamol or antibiotics (28 missing data)

${ }^{\mathrm{b}}$ Missing data for 15 subjects

mtDNA methylation at specific loci, and lower CpG-specific methylation levels of CYP1A1 in placental tissue.

Despite a limited number of (epi)genomic studies in placental tissue and cord blood, we are improving our understanding of the molecular pathways underlying the association between gestational tobacco smoke exposure and low birth weight. Combining gene expression and epigenome-wide methylation arrays Suter et al. [26] showed that the expression of 623 genes and the methylation of 1024 CpG dinucleotides were significantly altered in placentas of smokers. For 438 genes significant correlations were revealed between methylation and gene expression, and their potential functions or mechanisms were explored using an Ingenuity Pathway Analysis. The authors found that the gene list was enriched for genes involved in functional pathways such as mitochondrial dysfunction, oxidative phosphorylation and hypoxia. Indeed, mitochondria, the "powerhouses" of cells, provide cellular energy via oxidative phosphorylation and are very sensitive to exposures that induce oxidative stress. The double stranded circular mtDNA, of which multiple copies are present in mitochondria, is vulnerable to reactive oxygen species (ROS) because of an inefficient DNA repair capacity and close proximity to the 


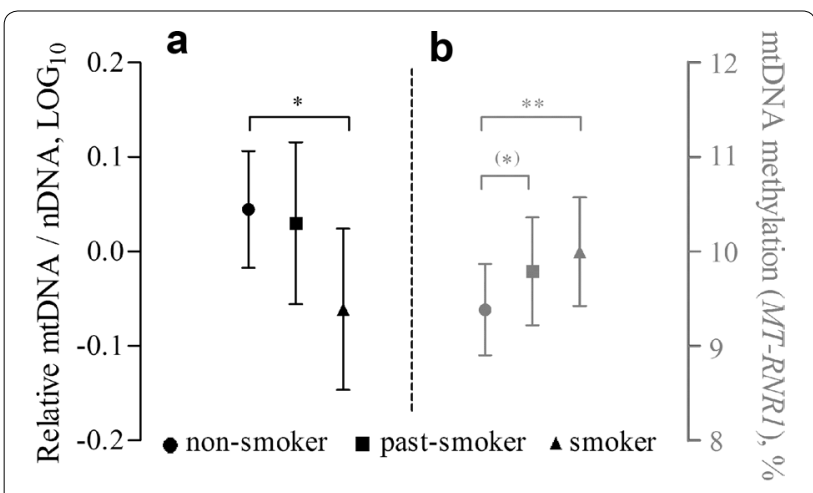

Placental mtDNA Placental mtDNA content methylation

Fig. 1 Estimated mean levels of mtDNA content and mtDNA methylation in placental tissue of non-smokers $(n=255)$, past smokers $(n=65)$, and current smokers $(n=62)$. The bars represent the estimated means with $95 \%$ confidence intervals for the non-smoking (filled circle), past-smoking (filled square), and smoking group (filled triangle). a Relative mtDNA content levels (unitless) are $\log _{10}$-transformed; $\mathbf{b}$ Methylation of the MT-RNR1 gene are absolute methylation levels. Both the generalized linear model for mtDNA content and the mixed-effects model for mtDNA methylation were adjusted for maternal age, gestational age, newborn's sex, maternal education, ethnicity, parity, pre-pregnancy BMl, and alcohol consumption. $\left.{ }^{*}\right) p=0.06 ;{ }^{*} p<0.05 ;{ }^{* *} p<0.005$ : difference compared to the nonsmoking group

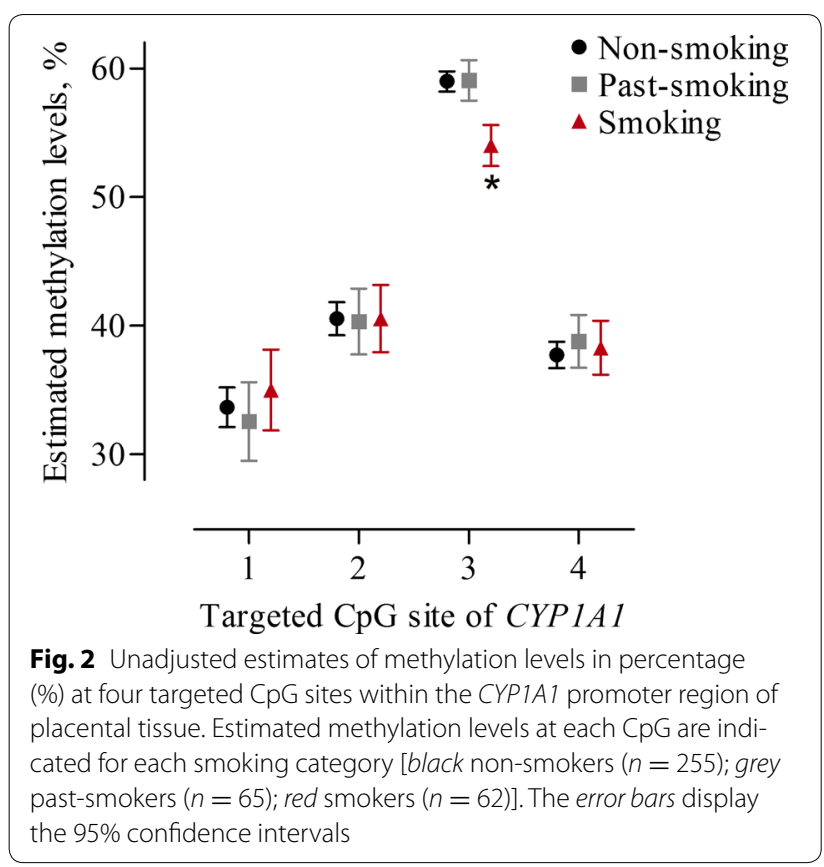

electron transport chain [33]. The estimated mutation rate of mtDNA is 5-10 times higher compared to nuclear DNA [34]. We showed that placental mtDNA content and methylation levels were responsive to tobacco smoke exposure during pregnancy indicating that mtDNA is a sensitive marker of mitochondrial damage and dysfunction as proposed by Sahin et al. [10]. In addition to other studies reporting changes in placental mtDNA content in smoking mothers $[7,8]$ or mothers exposed to air pollution [29], we provide here the first epidemiological evidence of altered methylation levels at specific loci of the mitochondrial genome of placental tissue in response to tobacco smoke exposure during pregnancy. We suggest that pollution-induced epigenetic modifications of the mitochondrial genome may prime alterations in mtDNA content by regulating mitochondrial function and biogenesis [35]. Damaged or non-functioning mitochondria are specifically degraded through mitophagy and could result in a depletion of mtDNA [36], which moreover may lead to changes in methylation patterns of a number of nuclear genes [12]. The sensitivity analysis showed that mtDNA content and mtDNA methylation correlated with methylation of $C Y P 1 A 1$ in placental tissue, which could be indicative of a relationship between mitochondrial dysfunction and the epigenetic landscape of the nuclear genome [11]. Whether mitochondrial dysfunction affects gene expression and methylation patterns of other genes needs to be elucidated.

An expanding body of evidence suggests that the epigenome of placental tissue and cord blood is sensitive to environmental exposures [13]. Epigenome-wide methylation studies are used to examine the epigenetic status of the human genome at many different loci in a number of individuals and also to assess whether any of these CpG loci are associated with a trait or an environmental pollutant [37]. A $450 \mathrm{~K}$ epigenome-wide methylation study by Joubert et al. [23] demonstrated differentially methylated detoxifying genes $(A H R R$ and $C Y P 1 A 1)$ in cord blood of newborns exposed to tobacco smoke during pregnancy. This finding was confirmed in another population of infants by analyzing whole blood obtained by a heel prick [25]. Maternal smoking as assessed by both self-report and cotinine levels in plasma showed higher methylation levels at different CpGs of CYP1A1 in cord blood [23]. Conversely, in placental tissue of smoker mothers, Suter et al. [19] observed hypomethylated CpG dinucleotides proximal to a xenobiotic response element (XRE); whereas, those distal from such elements did not demonstrate differential methylation. The authors calculated the total percentage of methylation for a distinct region of the promoter $(-1411$ to -1295 bp from the transcription start site) without taking into account the separate CpGs, unlike we did in our study. We observed lower methylation levels at a specific CpG site that lies adjacent to a XRE site in placental tissue of mothers who smoked during pregnancy. It is important to note that 
Table 2 Effect of tobacco smoking status during pregnancy on CpG sites of CYP1A1 in placental tissue $(n=382)$

\begin{tabular}{|c|c|c|c|c|c|c|c|}
\hline \multirow[t]{2}{*}{ CYP1A1 methylation ${ }^{a}$} & \multirow[t]{2}{*}{ Non-smoking } & \multicolumn{3}{|c|}{ Past-smoking } & \multicolumn{3}{|c|}{ Smoking } \\
\hline & & $\%$ & $(95 \% \mathrm{Cl})$ & $p$ value & $\%$ & $(95 \% \mathrm{Cl})$ & $p$ value \\
\hline CpG 1 & Ref. & -1.16 & $(-5.90$ to 3.56$)$ & 0.99 & 1.75 & $(-3.09$ to 6.60$)$ & 0.95 \\
\hline CpG 2 & Ref. & -0.30 & $(-4.25$ to 3.66$)$ & 0.99 & 0.43 & $(-3.63$ to 4.49$)$ & 0.99 \\
\hline CpG 3 & Ref. & 0.02 & $(-2.47$ to 2.51$)$ & 0.99 & -4.57 & $(-7.15$ to -1.98$)$ & $<0.0001$ \\
\hline CpG 4 & Ref. & 1.00 & $(-2.11$ to 4.11$)$ & 0.98 & 0.98 & $(-2.22$ to 4.19$)$ & 0.99 \\
\hline
\end{tabular}

Data shown in italic is significant

Mixed-effects models are adjusted for maternal age, gestational age, newborn's sex, maternal education, ethnicity, parity, pre-pregnancy BMI, and alcohol consumption

${ }^{\text {a }}$ Estimated absolute percentage (\%) change in methylation levels for each $\mathrm{CpG}$ of $\mathrm{CYP} 1 \mathrm{~A} 1$ compared to the non-smoking group (reference). The $95 \% \mathrm{Cl}$ and $p$ values are adjusted according to Dunnett's procedure
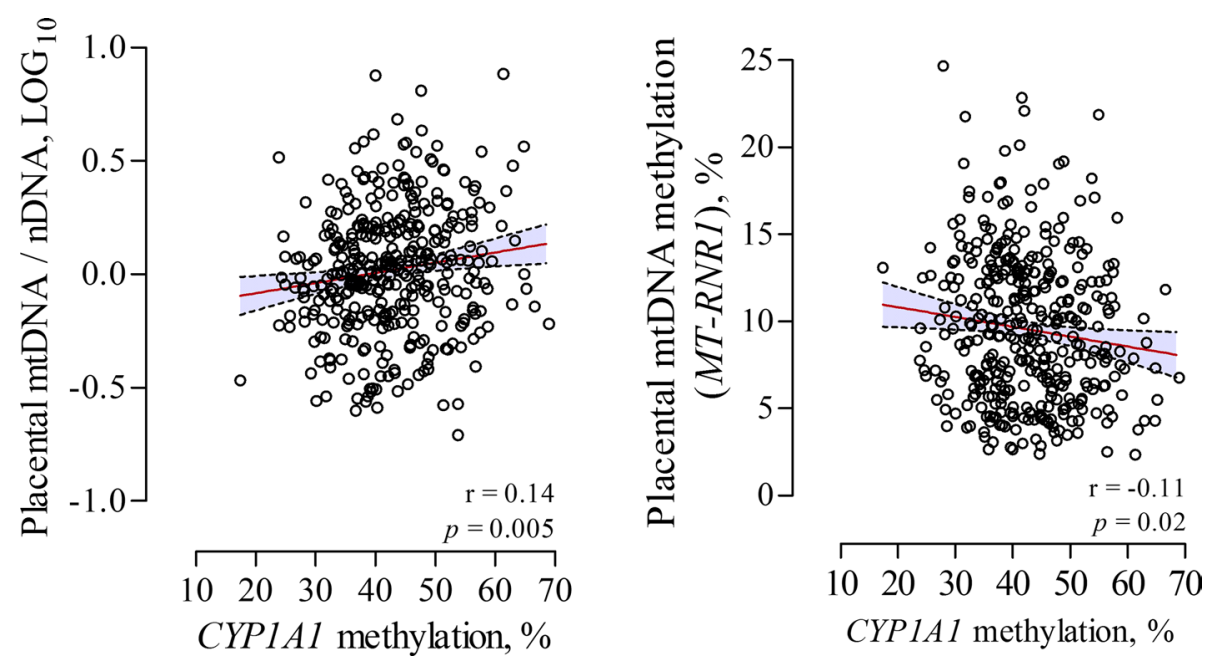

Fig. 3 Correlation between CYP1A1 methylation levels (\%) and mtDNA content ( $\left.\log _{10}\right)$ or mtDNA methylation (MT-RNR1) (\%) in placental tissue. The dashed lines in the correlation plots depict the $95 \% \mathrm{Cl}$

this specific $\mathrm{CpG}$ site harbors a $\mathrm{C} / \mathrm{G}$ single nucleotide polymorphism (SNP: rs3809585 with allele frequencies C: $1.717 \%$ and G: $98.283 \%)$. We are confident that this SNP did not affect DNA methylation since all pyrograms confirmed a $G$ nucleotide in the analyzed sequence. Interestingly, the study of Joubert et al. [23] in cord blood, the study of Suter et al. [19] in placental tissue, and our study in placental tissue, examined approximately the same region of interest and CpGs, however with different detection methods (Fig. 4). With the bisulfite pyrosequencing approach, we confirmed hypomethylation at a specific $C \mathrm{pG}$ of the $C Y P 1 A 1$ gene in placental tissue which is in contrast with the findings in cord blood [23]. Although we lacked meaningful gene expression data of CYP1A1 in our study, Suter et al. [19] previously showed that lower methylation levels in a region covering the XRE site were correlated with increased expression of CYP1A1 in placental tissue. Moreover, other studies demonstrated increased CYP1A1 mRNA [38] and protein [39] expression in human placentas in response to tobacco smoke exposure. Constituents of tobacco smoke such as PAHs enter cells and are recognized by the aryl hydrocarbon receptor (Ahr) causing its translocation to the nucleus and the formation of a heterodimer with the Ahr nuclear translocator protein (ARNT). This complex binds to genes with a XRE within the promoter and initiates expression of detoxifying enzymes involved in phase I and II xenobiotic metabolism [40].

A limitation of our study is the chance of exposure misclassification. Information about maternal smoking during pregnancy was based on self-report and is not verifiable. A possibility to overcome this limitation is the determination of the cotinine concentration in plasma or urine of the mother. Nevertheless, previous studies demonstrated that this would not be superior to self-reported smoking habit in pregnant women [2]. We acknowledge the fact that we cannot fully exclude residual or unmeasured confounding by other factors that 


\section{CYP1A1 Chr $15(+)$}

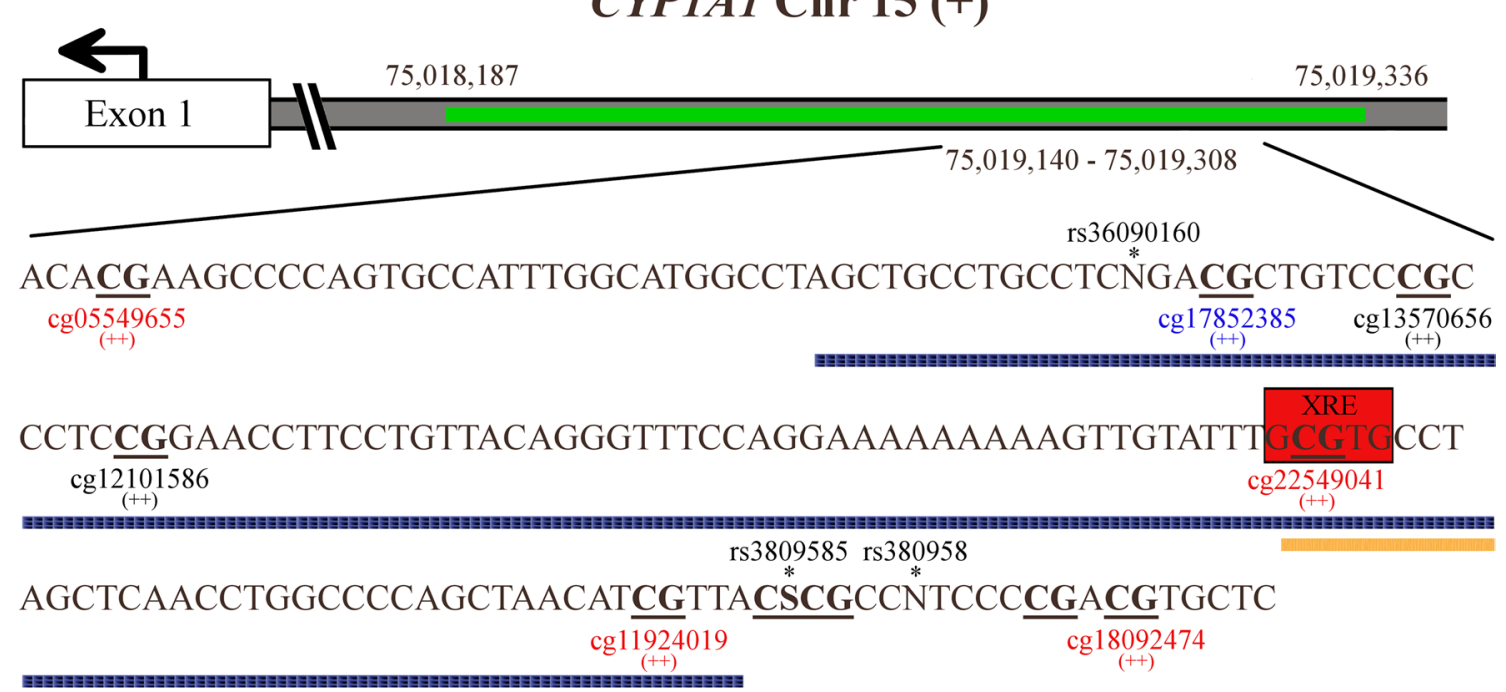

Fig. $4 \mathrm{CpG}$ sites located on the shore of a CpG island in a bidirectional regulatory region of the CYP1A1 gene. The CpG island is depicted in green with a distinct portion magnified (chr15:75,019,140-75,019,308). CpG sites are denoted in bold and underlined whereas possible SNPs are indicated with an asterisk. The orange bar represents the analyzed sequence in our study and includes four $\mathrm{CpG}$ sites. The blue bar represents the analyzed sequence in placental tissue derived from the article of Suter et al. [19] and includes five CpG sites. The cg probes that were investigated in the $450 \mathrm{~K}$ study of Joubert et al. [23] in cord blood are displayed with the color representing the statistical significance of the association between plasma cotinine and methylation of the probe (blue $p>1 \times 10^{-5}$; black $1 \times 10^{-5} \geq p \geq 1 \times 10^{-7}$; red $p<1 \times 10^{-7}$ ) and the magnitude of effect (++: higher methylation). The information on the figure is based on the UCSC Genome Browser on Human Feb. 2009, GRCh37/hg19

could be associated with both tobacco smoke exposure and placental molecular signatures. Although a causal relationship exists between prenatal tobacco smoke exposure and low birth weight or preterm birth, not all infants exposed to tobacco smoke develop these adverse perinatal outcomes. It is therefore reasonable to assume that several interactions exists between tobacco smoke exposure and biochemical, genetic, and epigenetic factors which make the fetus more susceptible to changes in fetal programming.

Our findings are of clinical relevance because responses of mitochondrial DNA and changes in the fetal methylome are plausible alterations that may underlie the adverse effect of tobacco smoke exposure on birth weight. They increase our knowledge on the mechanisms of perturbations in the fetoplacental exchange that might lie at basis of low birth weight and, hence, may be used in the broader sense of clinical context.

\section{Conclusions}

This study provides epidemiological evidence of molecular changes in placental tissue that can serve as molecular signatures of exposure to tobacco smoke during pregnancy. Whether the molecular signatures described in our study may be related to early developmental changes in Belgian children will be investigated in the ongoing follow-up study of the ENVIRONAGE birth cohort.

\section{Additional file}

Additional file 1. Additional table and figure.

\section{Abbreviations}

ACTB: beta actin; Ahr: aryl hydrocarbon receptor; Cl: confidence interval; CYP1A1: cytochrome P450, family 1, subfamily A, polypeptide 1; D-loop: displacement loop; ENVIRONAGE: ENVIRonmental influence ON early AGEing; MT-ND1: mitochondrial encoded NADH dehydrogenase 1; MTF3212/R3319: mitochondrial forward primer from nucleotide 3212 and reverse primer from nucleotide 3319; MT-RNR1: mitochondrial region RNR1; mtDNA: mitochondrial DNA; PAH: polycyclic hydrocarbon; qPCR: quantitative real-time polymerase chain reaction; RPLPO: acidic ribosomal phosphoprotein PO.

\section{Authors' contributions}

TSN coordinates the ENVIRONAGE birth cohort and designed the current study together with BGJ and AAB. WG and BGJ gave guidance to the midwives and did the quality control of the database. BGJ performed the experiments with the help of $\mathrm{HMB}$, and BGJ carried out statistical analysis. BGJ, $\mathrm{HMB}$, $A A B$, and TSN did the interpretation of the data. BGJ wrote the first draft of the manuscript. All authors read and approved the final manuscript.

\section{Author details}

${ }^{1}$ Centre for Environmental Sciences, Hasselt University, Hasselt, Belgium. ${ }^{2}$ Department of Obstetrics, East-Limburg Hospital, Genk, Belgium. ${ }^{3}$ Department of Physiology, Hasselt University, Diepenbeek, Belgium. ${ }^{4}$ Laboratory of Environmental Epigenetics, Exposure Epidemiology and Risk Program, Harvard School of Public Health, Boston, MA 02215, USA. ${ }^{5}$ Louvain Centre for Toxicology and Applied Pharmacology (LTAP), Université Catholique de Louvain, Brussels, Belgium. ${ }^{6}$ Department of Environmental Health Sciences, Mailman School of Public Health, Columbia University, New York, NY 10032, USA. ${ }^{7}$ Department of Public Health \& Primary Care, Occupational 
and Environmental Medicine, Leuven University, Louvain, Belgium. ${ }^{8}$ Centre for Environmental Sciences, Hasselt University, Agoralaan Gebouw D, 3590 Diepenbeek, Belgium.

\section{Acknowledgements}

The authors thank the participating mothers and neonates, as well as the staff of the maternity ward, midwives, and the staff of the clinical laboratory of EastLimburg Hospital in Genk.

\section{Competing interests}

The authors declare that they have no competing interests.

\section{Availability of data and materials}

The datasets used and/or analyzed during the current study are available from the corresponding author on reasonable request.

\section{Ethics approval and consent to participate}

All procedures were in line with the principles of the Helsinki Declaration for investigation of human subjects and approved by the Ethical Committee of Hasselt University and the East-Limburg Hospital. All participants provided written informed consent.

\section{Funding}

The ENVIRONAGE birth cohort is supported by the European Research Council (ERC-2012-StG.310898), by the Flemish Scientific Fund (FWO, G.0.733.15.N) and the Special Research Fund (BOF) of Hasselt University. This work was also supported by funding from the US National Institute of Environmental Health Sciences (R21ES022694 and R01ES021733).

Received: 8 November 2016 Accepted: 18 December 2016 Published online: 04 January 2017

\section{References}

1. Windham GC, Hopkins B, Fenster L, Swan SH. Prenatal active or passive tobacco smoke exposure and the risk of preterm delivery or low birth weight. Epidemiology. 2000;11(4):427-33.

2. Jaddoe VW, Troe EJ, Hofman A, Mackenbach JP, Moll HA, Steegers EA, et al. Active and passive maternal smoking during pregnancy and the risks of low birthweight and preterm birth: the Generation R Study. Paediatr Perinat Epidemiol. 2008;22(2):162-71.

3. Fantuzzi G, Aggazzotti G, Righi E, Facchinetti F, Bertucci E, Kanitz S, et al. Preterm delivery and exposure to active and passive smoking during pregnancy: a case-control study from Italy. Paediatr Perinat Epidemiol. 2007;21(3):194-200.

4. Cox B, Martens E, Nemery B, Vangronsveld J, Nawrot TS. Impact of a stepwise introduction of smoke-free legislation on the rate of preterm births: analysis of routinely collected birth data. BMJ. 2013;346:f441.

5. Jauniaux E, Burton GJ. Morphological and biological effects of maternal exposure to tobacco smoke on the feto-placental unit. Early Hum Dev. 2007;83(11):699-706.

6. US Department of Health and Human Service. How tobacco smoke causes disease-the biology and behavioral basis for tobacco-attributable disease: a report of the surgeon general 2010. http://www.surgeongeneral.gov/ library/reports/tobaccosmoke/full_report.pdf. Accessed 5 Oct 2016.

7. Bouhours-Nouet N, May-Panloup P, Coutant R, de Casson FB, Descamps $P$, Douay $O$, et al. Maternal smoking is associated with mitochondrial DNA depletion and respiratory chain complex III deficiency in placenta. Am J Physiol Endocrinol Metab. 2005;288(1):E171-7.

8. Garrabou G, Hernàndez A-S, Catalán García M, Morén C, Tobías E, Córdoba S, et al. Molecular basis of reduced birth weight in smoking pregnant women: mitochondrial dysfunction and apoptosis. Addict Biol. 2016:21(1):159-70.

9. Lee HC, Lu CY, Fahn HJ, Wei YH. Aging- and smoking-associated alteration in the relative content of mitochondrial DNA in human lung. FEBS Lett. 1998:441(2):292-6.

10. Sahin E, Colla S, Liesa M, Moslehi J, Muller FL, Guo M, et al. Telomere dysfunction induces metabolic and mitochondrial compromise. Nature. 2011;470(7334):359-65.
11. Minocherhomji S, Tollefsbol TO, Singh KK. Mitochondrial regulation of epigenetics and its role in human diseases. Epigenetics. 2012;7(4):326-34

12. Smiraglia DJ, Kulawiec M, Bistulfi GL, Gupta SG, Singh KK. A novel role for mitochondria in regulating epigenetic modification in the nucleus. Cancer Biol Ther. 2008;7(8):1182-90.

13. Vaiserman A. Epidemiologic evidence for association between adverse environmental exposures in early life and epigenetic variation: a potential link to disease susceptibility? Clin Epigenetics. 2015;7(1):96.

14. Urdinguio RG, Torró MI, Bayón GF, Álvarez-Pitti J, Fernández AF, Redon P, et al. Longitudinal study of DNA methylation during the first 5 years of life. J Transl Med. 2016;14:160.

15. Ivorra C, Fraga M, Bayon G, Fernandez A, Garcia-Vicent C, Chaves F, et al. DNA methylation patterns in newborns exposed to tobacco in utero. J Transl Med. 2015;13(1):25

16. Wilhelm-Benartzi CS, Houseman EA, Maccani MA, Poage GM, Koestler DC, Langevin SM, et al. In utero exposures, infant growth, and DNA methylation of repetitive elements and developmentally related genes in human placenta. Environ Health Perspect. 2011;120(2):296-302.

17. Michels KB, Harris HR, Barault L. Birthweight, maternal weight trajectories and global dna methylation of LINE-1 repetitive elements. PLoS ONE. 2011;6(9):e25254.

18. Guerrero-Preston R, Goldman LR, Brebi-Mieville P, Ili-Gangas C, Lebron C, Witter FR, et al. Global DNA hypomethylation is associated with in utero exposure to cotinine and perfluorinated alkyl compounds. Epigenetics. 2010;5(6):539-46.

19. Suter M, Abramovici A, Showalter L, Hu M, Shope CD, Varner M, et al. In utero tobacco exposure epigenetically modifies placental CYP1A1 expression. Metabolism. 2010;59(10):1481-90.

20. Bouwland-Both M, van Mil N, Tolhoek C, Stolk L, Eilers P, Verbiest M, et al. Prenatal parental tobacco smoking, gene specific DNA methylation, and newborns size: the Generation R study. Clin Epigenetics. 2015;7(1):83.

21. Novakovic B, Ryan J, Pereira N, Boughton B, Craig JM, Saffery R. Postnatal stability, tissue, and time specific effects of AHRR methylation change in response to maternal smoking in pregnancy. Epigenetics. 2014;9(3):377-86

22. Murphy SK, Adigun A, Huang Z, Overcash F, Wang F, Jirtle RL, et al. Gender-specific methylation differences in relation to prenatal exposure to cigarette smoke. Gene. 2012;494(1):36-43.

23. Joubert BR, Haberg SE, Nilsen RM, Wang X, Vollset SE, Murphy SK, et al. $450 \mathrm{~K}$ epigenome-wide scan identifies differential DNA methylation in newborns related to maternal smoking during pregnancy. Environ Health Perspect. 2012;120(10):1425-31.

24. Küpers LK, Xu X, Jankipersadsing SA, Vaez A, la Bastide-van Gemert S, Scholtens $S$, et al. DNA methylation mediates the effect of maternal smoking during pregnancy on birthweight of the offspring. Int J Epidemiol. 2015;44(4):1224-37.

25. Markunas CA, Xu Z, Harlid S, Wade PA, Lie RT, Taylor JA, et al. Identification of DNA methylation changes in newborns related to maternal smoking during pregnancy. Environ Health Perspect. 2014;122(10):1147-53.

26. Suter M, Ma J, Harris AS, Patterson L, Brown KA, Shope C, et al. Maternal tobacco use modestly alters correlated epigenome-wide placental DNA methylation and gene expression. Epigenetics. 2011;6(11):1284-94.

27. Januar V, Desoye G, Novakovic B, Cvitic S, Saffery R. Epigenetic regulation of human placental function and pregnancy outcome: considerations for causal inference. Am J Obstet Gynecol. 2015;213(4, Supplement):S182-96.

28. Janssen BG, Madlhoum N, Gyselaers W, Bijnens E, Clemente DB, Cox $B$, et al. Cohort profile: the ENVIRonmental influence ON early AGEing (ENVIRONAGE): a Birth Cohort Study. Int J Epidemiol. 2016. doi:10.1093/ ije/dyw269.

29. Janssen BG, Munters E, Pieters N, Smeets K, Cox B, Cuypers A, et al. Placental mitochondrial DNA content and particulate air pollution during in utero life. Environ Health Perspect. 2012;120(9):1346-52.

30. Janssen BG, Byun H-M, Cox B, Gyselaers W, Izzi B, Baccarelli AA, et al. Variation of DNA methylation in candidate age-related targets on the mitochondrial-telomere axis in cord blood and placenta. Placenta. 2014;35(9):665-72.

31. Hellemans J, Mortier G, De Paepe A, Speleman F, Vandesompele J. qBase relative quantification framework and software for management and 
automated analysis of real-time quantitative PCR data. Genome Biol 2007;8(2):R19

32. Valeri L, Vanderweele TJ. Mediation analysis allowing for exposuremediator interactions and causal interpretation: theoretical assumptions and implementation with SAS and SPSS macros. Psychol Methods. 2013;18(2):137-50.

33. Linnane AW, Marzuki S, Ozawa T, Tanaka M. Mitochondrial DNA mutations as an important contributor to ageing and degenerative diseases. Lancet. 1989; 1 (8639):642-5.

34. Payne BAI, Wilson IJ, Yu-Wai-Man P, Coxhead J, Deehan D, Horvath R, et al. Universal heteroplasmy of human mitochondrial DNA. Hum Mol Genet. 2013;22(2):384-90.

35. Janssen BG, Byun H-M, Gyselaers W, Lefebvre W, Baccarelli AA, Nawrot TS. Placental mitochondrial methylation and exposure to airborne particulate matter in the early life environment: an ENVIRONAGE birth cohort study. Epigenetics. 2015;10(6):536-44.

36. Ding WX, Yin XM. Mitophagy: mechanisms, pathophysiological roles, and analysis. Biol Chem. 2012;393(7):547-64.
37. Michels KB, Binder AM, Dedeurwaerder S, Epstein CB, Greally JM, Gut I, et al. Recommendations for the design and analysis of epigenome-wide association studies. Nat Methods. 2013;10(10):949-55.

38. Wadzinski TL, Geromini K, McKinley Brewer J, Bansal R, Abdelouahab N, Langlois MF, et al. Endocrine disruption in human placenta: expression of the dioxin-inducible enzyme, CYP1A1, is correlated with that of thyroid hormone-regulated genes. J Clin Endocrinol Metab. 2014;99(12):E2735-43.

39. Sbrana E, Suter MA, Abramovici AR, Hawkins HK, Moss JE, Patterson L, et al. Maternal tobacco use is associated with increased markers of oxidative stress in the placenta. Am J Obstet Gynecol. 2011;205(3):246.e1-7.

40. Harper PA, Riddick DS, Okey AB. Regulating the regulator: factors that control levels and activity of the aryl hydrocarbon receptor. Biochem Pharmacol. 2006;72(3):267-79.

\section{Submit your next manuscript to BioMed Central and we will help you at every step:}

- We accept pre-submission inquiries

- Our selector tool helps you to find the most relevant journal

- We provide round the clock customer support

- Convenient online submission

- Thorough peer review

- Inclusion in PubMed and all major indexing services

- Maximum visibility for your research

Submit your manuscript at www.biomedcentral.com/submit 